\title{
Role of stemness-related molecules in neuroblastoma
}

\author{
Takehiko Kamijo'
}

Neuroblastoma (NB) is the most common pediatric solid malignant tumor derived from the sympathetic nervous system. High-risk NB is still one of the most difficult tumors to cure, with only 40\% long-term survival despite intensive multimodal therapy. The clinical presentation and treatment response of advanced NB, which results in relapse and a refractory state after a good response to the initial chemotherapy, suggests that cancer stem cells (CSCs) likely exist in NB tumors. Putative CSCs using primary tumor sphere formation from NB patients were reported previously, and several molecules will be elucidated from the tumor sphere to develop CSC-targeting therapies. Recently, our group reported that a CSC marker for several malignancies, CD133, and the stemnessrelated polycomb BMI1 have functions to repress NB cell differentiation. Depletion of CD133 or BMI1 effectively induced neurite elongation and marker molecules for differentiation in NB cells. Of note, CD133-related NB cell differentiation and RET (rearranged during transfection) repression were considerably dependent on p38MAPK and phosphoinositide 3-kinase (PI3K)/AKT pathways. Intriguingly, both CD133 and BMI1 also have a role in xenograft tumor formation and tumor sphere formation. These observations suggest that CD133 and BMI1 may be candidates for the development of CSC-targeting therapies for refractory NB patients.

\section{CANCER STEM CELLS (CSCS)}

In recent years, accumulating experimental evidence has suggested that tumors have a hierarchal organization regulated by a minority of cells, the CSCs (1-3). CSCs are defined as "a small subset of cancer cells within a cancer that constitute a reservoir of self-sustaining cells with the exclusive ability to self-renew and to cause the heterogeneous lineages of cancer cells that comprise the tumor" (4). The concept of CSCs has immediate therapeutic consequences: if cancer growth is sustained by CSCs, then curative therapy will require targeting of this specific subpopulation (5). The CSC model proposes that the growth and progression of many cancers are driven by small subpopulations of CSCs. This model does not address the question of whether cancers arise from normal stem cells. Instead, it suggests that irrespective of the cell origin, many cancers may be hierarchically organized in a similar manner to normal tissues. It is argued that just as normal stem cells differentiate into phenotypically diverse progeny with limited proliferative potential, CSCs also undergo epigenetic changes analogous to the differentiation of normal cells, forming phenotypically diverse nontumorigenic cancer cells that compose the bulk of cells in a tumor. The term "tumor-initiating cells (TICs)" has been used almost synonymously with "CSCs" in many reports, although "TIC" was used when researchers placed emphasis on the exclusive ability to self-renew and to constitute tumors. Compelling data support the CSC model in various human malignancies, including malignant germ cell tumors (6), leukemias (7), breast cancers (8), brain tumors (9), and colon cancers $(10,11)$.

The concept that tumorigenic cells can be distinguished from nontumorigenic cells on the basis of surface marker expression is fundamental to the CSC model. In the above-mentioned tumors, the CSC markers were identified, e.g., leukemia (CD34 ${ }^{+}$ $\left.\mathrm{CD} 38^{-}\right)$, brain tumors $\left(\mathrm{CD} 133^{+}\right)$, colon cancers $\left(\mathrm{CD} 133^{+}\right.$, EpCAM high $\left.\mathrm{CD}_{4} 4^{+}, \mathrm{ALDH}^{+}\right)$, breast cancers $\left(\mathrm{CD} 44^{+} \mathrm{CD} 24^{-}\right.$, $\mathrm{ALDH}^{+}$). Because markers will be further evaluated in additional studies and in a larger numbers of tumors, some markers will likely prove less robust than they currently appear. For example, initial work on brain tumors identified CD133 as a robust marker of CSCs (11); however, additional studies have shown that CD133 identifies CSCs in some specific brain tumor subtypes rather than all subtypes (12). Similar results have been emerging with colon cancer; combinations and permutations of CD133, CD44, and aldehyde dehydrogenase-1 (ALDH1) have been studied with conflicting results about which marker or combination thereof best identifies the CSC population $(11,13)$. Given the uncertainty of CSC marker robustness, markers alone should not be relied on to assess potential biological differences between tumorigenic and nontumorigenic cells; functional assays are required to confirm differences in therapy sensitivity and other biological properties.

\section{Neuroblastoma (NB)}

NB is an extracranial pediatric solid tumor that remains difficult to cure in advanced stages. NBs are neuroectodermal 
tumors of embryonic neural crest-derived cells. The neural crest in normal development gives rise to nerve cells of the sympathetic nervous system. The fetal adrenal medulla consists of a mixture of chromaffin cells and clusters of mature ganglion cells; NBs most likely originate from a pluripotent precursor cell or from both cell types because NB tumors can contain cells with both neuronal and chromaffin cell phenotypes. From these observations, it is widely assumed that NBs are embryonal tumors. This means that they are considered to originate from a developmental defect that prevents normal cellular differentiation and locks cells in a state of increased growth (14).

In the group of NBs, different risk categories can be identified: patients with high-, intermediate-, or low-risk tumors. Highrisk tumors include disseminated disease or bulky tumors with typical genetic alterations, such as amplification of $N-M Y C$ (INSS (International Neuroblastoma Staging System) stages 3 and 4). Intermediate-risk diseases are characterized by large, unresectable, localized tumors without any metastasis (INSS stages $2 b-3$ ). Low-risk NBs include small tumors (INSS stages $1-2 \mathrm{a})$, which can be treated by surgery alone and will lead to an excellent 5-y event-free survival (EFS) of more than $90 \%$ (15). One more category is formed by infants (below $1 \mathrm{y}$ of age at diagnosis) with a small primary tumor and dissemination. The dissemination is according to a limited and characteristic pattern involving the liver, bone marrow, and/or skin, but not bone (stage 4s); these patients have an excellent 5-y EFS of 70 to $90 \%$ and a high rate of spontaneous regression (16).

Overall prognosis of patients with NB has greatly improved, with 5 -y survival rates increasing from $52 \%$ during the period from 1975 through 1977 to $74 \%$ during the period from 1999 through 2005, according to the Surveillance, Epidemiology, and End Results databases (http://www.seer.cancer.gov). However, unlike the many childhood malignancies for which survival has been improved by recent therapies, high-risk NB is still one of the most difficult tumors to cure, with only $40 \%$ long-term survival despite intensive multimodal therapy (17).

The clinical presentation and treatment response of high-risk $\mathrm{NB}$, which results in relapse and a refractory state after a good response to the initial chemotherapy, suggest that CSCs likely exist in NB tumors. Furthermore, the origin of the above-mentioned NBs from a pluripotent precursor cell may support the existence of stem cell-like tumor cells in NB tumors. Recently, the Children's Oncology Group conducted a study to determine whether adding ch14.18, GM-CSF, and interleukin-2 to standard isotretinoin therapy after intensive multimodal therapy would improve outcomes in high-risk NB and found that immunotherapy with ch14.18, GM-CSF, and interleukin-2 was associated with a significantly improved outcome as compared with standard therapy in patients with high-risk NB. Immunotherapy was superior to standard therapy with regard to rates of EFS ( $66 \pm 5 \%$ vs. $46 \pm 5 \%$ at $2 y, P=0.01$ ) and overall survival ( $86 \pm 4 \%$ vs. $75 \pm 5 \%$ at $2 y, P=0.02)$ (ref. 18). Again, these observations confirm the clinical significance of CSCs in NB that are resistant to intensive multimodal therapy. NB CSCs may escape treatment and contribute to tumor relapse, therefore new therapies that target CSCs may prevent or treat tumor recurrences.

\section{CSC MODEL AND THERAPEUTIC APPROACHES IN NB}

The heterogeneity of NB tumor histology, which suggests the existence of a self-renewing multipotent CSC in NB, was partially addressed in a study by Ross's group (19). This I-type cell is a small, flattened, moderately adherent cell, with or without neuritic processes, which forms aggregates in culture. This cell type appears to represent a more primitive stem cell, a progenitor of N- or S-type cells, capable both of self-renewal and bidirectional differentiation (20). I-type cells are significantly more malignant than $\mathrm{N}$ - or S-type cells, with four- to fivefold greater plating efficiencies in soft agar and sixfold higher tumorigenicity in athymic mice. Furthermore, a CSC-related marker CD133 was highly expressed in I-type but not in $\mathrm{N}$ - and S-type NB cells, suggesting the role of CD133 in the stem cell-like phenotypes in I-type cells.

Recently, Kaplan's group indicated that dissociated cells from tumors or bone marrow grew as spheres in conditions used to culture neural crest stem cells, were capable of self-renewal, and exhibited chromosomal aberrations typical of NB. Primary spheres from all tumor risk groups differentiated under neurogenic conditions to form neurons. Impressively, as few as 10 passaged tumor sphere cells from aggressive NB injected orthotopically into severe combined immunodeficient/Beige mice formed large NB tumors that metastasized to several organs. Furthermore, highly tumorigenic tumor spheres were isolated from the bone marrow of patients in clinical remission, suggesting that this population of cells may predict clinical behavior and serve as a biomarker for minimal residual disease in high-risk patients (21).

The researchers extended their work and identified compounds that selectively target patient-derived CSC-like TICs while sparing normal pediatric stem cells (skin-derived precursors, SKPs) and characterized two therapeutic candidates. DECA-14 and rapamycin were identified as NB TIC-selective agents. Both compounds induced TIC death at nanomolar concentrations in vitro, significantly reduced NB xenograft tumor weight in vivo, and markedly decreased self-renewal or tumor-initiation capacity in treated tumors (22).

Furthermore, to identify signaling pathways important for the survival and self-renewal of NB TICs and potential therapeutic targets, they screened a small-molecule library of 143 protein kinase inhibitors, including 33 in clinical trials (23). Cytostatic or cytotoxic drugs were identified that targeted phosphoinositide 3-kinase (PI3K)/Akt, PKC (protein kinase C), Aurora, ErbB2, Trk, and Polo-like kinase 1 (PLK1). Treatment with PLK1 siRNA or low nanomolar concentrations of BI 2536 or BI 6727, PLK1 inhibitors in clinical trials for adult malignancies, were cytotoxic to TICs, whereas only micromolar concentrations of the inhibitors were cytotoxic for normal pediatric neural stem cells. Furthermore, BI 2536 significantly inhibited TIC tumor growth in a therapeutic xenograft model, both as a single agent and in combination with irinotecan, an active agent for relapsed NB. Together, PLK1 
may be a candidate kinase that regulates TIC growth and survival, and PLK1 inhibitors seem to be promising candidates as therapy for metastatic NB.

\section{Role of CD133 in NB Stemness}

CD133 (prominin-1) was the first identified member of the prominin family of cell-surface glycoproteins harboring five transmembrane domains (24). The specific functions and ligands of the prominins are still relatively unclear, but they are distinct in their restricted expression within plasma membrane protrusions, such as epithelial microvilli and epididymal ductal epithelial sterocilia. Regarding the function of CD133, Maw et al. (25) reported homozygosity for a 1-bp deletion (1878delG) in exon 16 of the CD133 gene predicted to cause a frameshift at codon 614 and a prematurely truncated protein lacking about half of the second extracellular loop, the final membrane-spanning segment, and the cytoplasmic C-terminal domain; this missense mutation caused retinal degeneration in four affected members of a consanguineous Indian family. This finding was further confirmed by an article demonstrating that the loss of Prom-1 in genetically modified mice results in the progressive degeneration of mature photoreceptors with complete loss of vision (26). Studies have now confirmed the utility of CD133 as a marker of hematopoietic stem cells for human allogeneic transplantation (27). In addition, CD133 represents a marker of TICs in a number of human cancers, e.g., brain tumors (9), colon cancers $(10,11)$, pancreatic cancers (28), and hepatocellular cancers (29), and therefore it may be possible to develop future therapies toward targeting CSCs via this marker.

A previous report indicated the isolation and characterization of putative TICs using primary sphere formation with tumors and bone marrow (BM) metastases from NB patients, although CD133 expression was not detected in a BM-derived high-risk NB tumor sphere sample (21). However, it was reported that subcloned NB cells (designated "I-type"), which have a significantly more malignant phenotype, with four- to fivefold greater plating efficiencies in soft agar and sixfold higher tumorigenicity in athymic mice, expressed high amounts of CD133 mRNA compared with less malignant subclones (19); therefore, the role of CD133 in NB tumorigenesis and aggressiveness remained unresolved.

To address the role of $\mathrm{CD} 133$ in $\mathrm{NB}$ tumorigenesis, we transduced CD133 cDNA or CD133-knocked-down shRNA by lentivirus vector in NB cell lines and primary NB tumor spheres (30). First, we knocked down CD133 in highly expressing NB cells and analyzed the knockdown-induced phenotype. CD133 knockdown in highly expressing NB cells effectively resulted in significant growth retardation in adherent cell culture, soft agar culture, and xenograft tumor formation in athymic mice. In accordance with this, ectopic CD133 expression in CD133-low NB cells accelerated proliferation and colony formation in soft agar. In CD133-knocked-down NB cells, neurite formation and GAP43/NF68 as neuronal differentiation markers were clearly upregulated. Expression analysis of the NB cell differentiation-related growth factor receptors/ ligands in CD133-expressed or CD133-reduced NB cells indicated that transcription of the protooncogene RET (rearranged during transfection) was suppressed by CD133. In addition, in $20 \mathrm{NB}$ cell lines and 12 unfavorable patient-derived primary NB tumors, RET expression was markedly repressed in CD133-expressing NB cells. CD133/RET coexpression cancelled the inhibition of NB cell differentiation by CD133, which was caused by CD133-related activation of p38MAPK and PI3K/Akt pathways (Figure 1). Intriguingly, CD133 knockdown resulted in inhibition of tumor sphere formation in both a NB cell line and primary tumor sphere-forming cells, suggesting that CD133 has a role in tumor cell stemness in NBs (Figure 1), which is consistent with a previous report describing that $\mathrm{CD} 133^{+}$cells showed increased sphere formation and tumorigenicity in tumor sphere-forming LAN5 NB cells (31).

CD133 was previously characterized as having five alternative promoters (P1-P5) that are active in a tissue-dependent manner (32). The P1, P2, and P3 promoters are located within a 1,540bp CpG island, whereas promoters P4 and P5 are not encompassed by $\mathrm{CpG}$-rich sequences. We will study the CD133 expression mechanism in $\mathrm{NB}$ cells, including CD133 upregulation in sphere-forming NB cells, because of the observation of the positive effect of CD133 on NB tumor sphere formation (30). In addition, we and others detected the $C D 133$ promoter regions mainly working in NB cells. We analyzed the important promoter regions for CD133 expression in tumor sphere-forming NB cells because a significant increase was observed in the NB tumor sphere (H. Takenobu and T. Kamijo, data not shown) (2). An increase in RNA and protein levels of CD133 was achieved following demethylation by assays using 5 -aza-2'-deoxycytidine (33). The significance of $C D 133$ promoter methylation in tumorigenesis in several tumors is still unresolved $(34,35)$.

\section{Role of BMI1 in NB Stemness}

In tumorigenesis, besides the well-known genetic changes that occur in cancer-such as the amplification/activation of oncogenes, deletion of tumor suppressor genes, and loss of

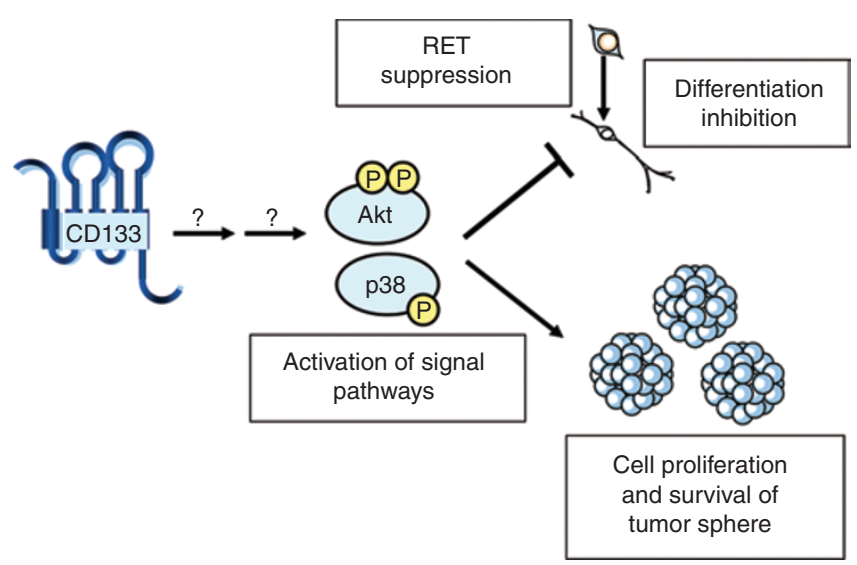

Figure 1. Role of CD133 in undifferentiated phenotypes of neuroblastoma cells. CD133 activates PI3K/AKT and p38MAPK pathways and maintains undifferentiated phenotypes of neuroblastoma cells. The cause of PI3K/AKT and p38MAPK pathway activation needs to be addressed. PI3K, phosphoinositide 3-kinase. 
heterozygosity or gene mutations in tumor-associated genes (36) - epigenetic alterations, such as altered DNA methylation, misregulation of chromatin remodeling by histone modifications, and aberrant expression of Polycomb group genes (PcGs) and trithorax group proteins, have emerged as common hallmarks of many cancers $(37,38)$. PcG proteins are epigenetic gene silencers that are implicated in neoplastic development. Their oncogenic function might be associated with their wellestablished role in the maintenance of embryonic and adult stem cells. Components of polycomb repressive complex 1 (PRC1; such as BMI1) (39) and PRC2 (such as EZH2) (40) are amplified and/or overexpressed in a broad spectrum of tumors, suggesting the roles of polycomb group proteins as oncogenes in several tumors.

Regarding BMI1 roles in NB, the binding of E2F-1 to BMI1 promoter and its activation were reported, and a strong expression of BMI1 was observed in primary NBs (41); however, BMI1 expression was not evaluated according to patient prognosis, and there was no correlation between MYCN amplification and BMI1 expression in the report. Another group reported that BMI1 knockdown induced the differentiation and growth suppression of NB cells, although BMI1 overexpression in NB cells could not function as an oncogenic stimulation (42). A recent report also suggested a role of BMI1 in NB tumorigenesis with the observation that a proliferation-specific transcriptional factor, FoxM1, regulates the differentiation and tumorigenesis of $\mathrm{NB}$ cells and is able to activate the expression of pluripotency gene Sox2 and $B M I 1$ in NB cells (43). The exact role of BMI1 in NB tumorigenesis has not been elucidated.

To study the role of BMI1 in NB tumorigenesis and its application to the development of molecular-targeted therapy, we analyzed the mechanism of BMI1 expression in NB and effect of BMI1 on NB cell functions (44). First, we studied BMI1 expression by western blotting and found that the PRC1 complex protein BMI1 expression correlated with MYCN protein expression in NB cell lines and primary NB tumors. BMI1 induction by MYCN was at the mRNA level in a MYCN-inducible NB cell line and several NB cell lines. BMI1 promoter analysis by luciferase vector experiments identified a MYCN-binding E-box sequence in the promoter region and chromatin immunoprecipitation experiments confirmed direct binding of MYCN around the E-box region. Next, we transduced BMI1 in several NB cell lines by lentivirus vectors and found upregulation of cell proliferation in vitro and in vivo. Consistent with this, BMI1 knockdown using shRNAs produced by lentivirus vectors resulted in the induction of neurite extension and the expression of differentiation markers GAP43 and NF68. To understand how BMI1 controls NB cell proliferation and differentiation, we chose to identify their target genes, except for $p 14 A R F / p 16 I N K 4 a$, as we could not observe significant changes in these wellknown tumor suppressors. To identify BMI1 target genes, except for p14ARF and p16INK4a, we studied expression gene profiling using an appropriate $\mathrm{NB}$ cDNA microarray (named the CCC-NHR13000 ChIP) carrying 13,440 cDNA

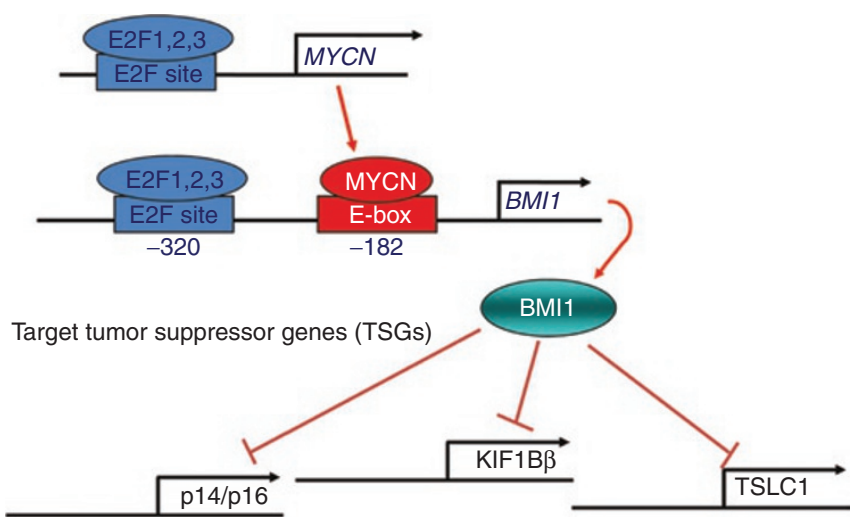

Figure 2. Epigenetic TSG suppression in NB tumorigenesis. MYCN upregulates BMI1 transcription and results in epigenetic suppression of several tumor suppressor genes (TSGs) in neuroblastoma (NB).

spots. Intriguingly, well-known NB tumor suppressor genes TSLC1 (NM_014333.3) and KIF1Bß (AB017133) are ranked as the first and second targets, respectively. We found that BMI 1 expression considerably repressed TSLC1 and KIF1B $\beta$ transcription in NB cells; by quantitative chromatin immunoprecipitation experiments, we showed that BMI1 specifically binds to the KIF1B $\beta$ (ENSG00000054523) and TSLC1 (ENSG00000105767) promoter regions in NB cells. Taken together, we found an intriguing MYCN/BMI1/tumor-suppressor pathway in NB cells (Figure 2). This pathway might have a marked impact on NB tumorigenesis and is considered to be a target for the development of molecular-targeted therapy for refractory NBs.

\section{ACKNOWLEDGMENTS}

The author thanks Daniel Mrozek, Medical English Service, for editorial assistance.

\section{STATEMENT OF FINANCIAL SUPPORT}

This work was supported in part by a grant-in-aid from the National Cancer Center Research and Development Fund (4).

\section{REFERENCES}

1. Reya T, Morrison SJ, Clarke MF, Weissman IL. Stem cells, cancer, and cancer stem cells. Nature 2001;414:105-11.

2. Dick JE. Stem cell concepts renew cancer research. Blood 2008;112: 4793-807.

3. Visvader JE, Lindeman GJ. Cancer stem cells in solid tumours: accumulating evidence and unresolved questions. Nat Rev Cancer 2008;8:755-68.

4. Clarke MF, Dick JE, Dirks PB, et al. Cancer stem cells-perspectives on current status and future directions: AACR Workshop on cancer stem cells. Cancer Res 2006;66:9339-44.

5. Tang C, Ang BT, Pervaiz S. Cancer stem cell: target for anti-cancer therapy. FASEB J 2007;21:3777-85.

6. Illmensee K, Mintz B. Totipotency and normal differentiation of single teratocarcinoma cells cloned by injection into blastocysts. Proc Natl Acad Sci USA 1976;73:549-53.

7. Bonnet D, Dick JE. Human acute myeloid leukemia is organized as a hierarchy that originates from a primitive hematopoietic cell. Nat Med 1997;3:730-7.

8. Al-Hajj M, Wicha MS, Benito-Hernandez A, Morrison SJ, Clarke MF Prospective identification of tumorigenic breast cancer cells. Proc Natl Acad Sci USA 2003;100:3983-8.

9. Singh SK, Hawkins C, Clarke ID, et al. Identification of human brain tumour initiating cells. Nature 2004;432:396-401. 
10. O’Brien CA, Pollett A, Gallinger S, Dick JE. A human colon cancer cell capable of initiating tumour growth in immunodeficient mice. Nature 2007;445:106-10.

11. Ricci-Vitiani L, Lombardi DG, Pilozzi E, et al. Identification and expansion of human colon-cancer-initiating cells. Nature 2007;445:111-5.

12. Joo KM, Kim SY, Jin X, et al. Clinical and biological implications of CD133-positive and CD133-negative cells in glioblastomas. Lab Invest 2008;88:808-15.

13. Huang EH, Hynes MJ, Zhang T, et al. Aldehyde dehydrogenase 1 is a marker for normal and malignant human colonic stem cells (SC) and tracks SC overpopulation during colon tumorigenesis. Cancer Res 2009;69:3382-9.

14. van Noesel MM, Versteeg R. Pediatric neuroblastomas: genetic and epigenetic 'danse macabre'. Gene 2004;325:1-15.

15. Brodeur GM. Neuroblastoma: biological insights into a clinical enigma. Nat Rev Cancer 2003;3:203-16.

16. Evans AE, D’Angio GJ, Randolph J. A proposed staging for children with neuroblastoma. Children's cancer study group A. Cancer 1971;27:374-8.

17. Maris JM, Hogarty MD, Bagatell R, Cohn SL. Neuroblastoma. Lancet 2007;369:2106-20.

18. Yu AL, Gilman AL, Ozkaynak MF, et al.; Children's Oncology Group. AntiGD2 antibody with GM-CSF, interleukin-2, and isotretinoin for neuroblastoma. N Engl J Med 2010;363:1324-34.

19. Walton JD, Kattan DR, Thomas SK, et al. Characteristics of stem cells from human neuroblastoma cell lines and in tumors. Neoplasia 2004;6:838-45.

20. Ross RA, Spengler BA, Domènech C, Porubcin M, Rettig WJ, Biedler JL. Human neuroblastoma I-type cells are malignant neural crest stem cells. Cell Growth Differ 1995;6:449-56.

21. Hansford LM, McKee AE, Zhang L, et al. Neuroblastoma cells isolated from bone marrow metastases contain a naturally enriched tumor-initiating cell. Cancer Res 2007;67:11234-43.

22. Smith KM, Datti A, Fujitani M, et al. Selective targeting of neuroblastoma tumour-initiating cells by compounds identified in stem cell-based small molecule screens. EMBO Mol Med 2010;2:371-84.

23. Grinshtein N, Datti A, Fujitani M, et al. Small molecule kinase inhibitor screen identifies polo-like kinase 1 as a target for neuroblastoma tumorinitiating cells. Cancer Res 2011;71:1385-95.

24. Corbeil D, Fargeas CA, Huttner WB. Rat prominin, like its mouse and human orthologues, is a pentaspan membrane glycoprotein. Biochem Biophys Res Commun 2001;285:939-44.

25. Maw MA, Corbeil D, Koch J, et al. A frameshift mutation in prominin (mouse)-like 1 causes human retinal degeneration. Hum Mol Genet 2000;9:27-34

26. Zacchigna S, Oh H, Wilsch-Bräuninger $\mathrm{M}$, et al. Loss of the cholesterolbinding protein prominin-1/CD133 causes disk dysmorphogenesis and photoreceptor degeneration. J Neurosci 2009;29:2297-308.

27. Bhatia M. AC133 expression in human stem cells. Leukemia 2001; 15:1685-8.
28. Hermann PC, Huber SL, Herrler T, et al. Distinct populations of cancer stem cells determine tumor growth and metastatic activity in human pancreatic cancer. Cell Stem Cell 2007;1:313-23.

29. Ma S, Tang KH, Chan YP, et al. miR-130b Promotes CD133(+) liver tumorinitiating cell growth and self-renewal via tumor protein 53-induced nuclear protein 1. Cell Stem Cell 2010;7:694-707.

30. Takenobu H, Shimozato O, Nakamura T, et al. CD133 suppresses neuroblastoma cell differentiation via signal pathway modification. Oncogene 2011;30:97-105.

31. Mahller YY, Williams JP, Baird WH, et al. Neuroblastoma cell lines contain pluripotent tumor initiating cells that are susceptible to a targeted oncolytic virus. PLoS ONE 2009;4:e4235.

32. Shmelkov SV, Jun L, St Clair R, et al. Alternative promoters regulate transcription of the gene that encodes stem cell surface protein AC133. Blood 2004;103:2055-61.

33. Schiapparelli P, Enguita-Germán M, Balbuena J, Rey JA, Lázcoz P, Castresana JS. Analysis of stemness gene expression and CD133 abnormal methylation in neuroblastoma cell lines. Oncol Rep 2010;24:1355-62.

34. Baba T, Convery PA, Matsumura N, et al. Epigenetic regulation of CD133 and tumorigenicity of $\mathrm{CD}_{133^{+}}$ovarian cancer cells. Oncogene 2009;28: 209-18.

35. Yi JM, Tsai HC, Glöckner SC, et al. Abnormal DNA methylation of CD133 in colorectal and glioblastoma tumors. Cancer Res 2008;68:8094-103.

36. Hanahan D, Weinberg RA. The hallmarks of cancer. Cell 2000;100: 57-70.

37. Sparmann A, van Lohuizen M. Polycomb silencers control cell fate, development and cancer. Nat Rev Cancer 2006;6:846-56.

38. Rajasekhar VK, Begemann M. Concise review: roles of polycomb group proteins in development and disease: a stem cell perspective. Stem Cells 2007;25:2498-510.

39. Valk-Lingbeek ME, Bruggeman SW, van Lohuizen M. Stem cells and cancer; the polycomb connection. Cell 2004;118:409-18.

40. Bracken AP, Pasini D, Capra M, Prosperini E, Colli E, Helin K. EZH2 is downstream of the $\mathrm{pRB}-\mathrm{E} 2 \mathrm{~F}$ pathway, essential for proliferation and amplified in cancer. EMBO J 2003;22:5323-35.

41. Nowak K, Kerl K, Fehr D, et al. BMI1 is a target gene of E2F-1 and is strongly expressed in primary neuroblastomas. Nucleic Acids Res 2006;34:1745-54

42. Cui H, Ma J, Ding J, Li T, Alam G, Ding HF. Bmi-1 regulates the differentiation and clonogenic self-renewal of I-type neuroblastoma cells in a concentration-dependent manner. J Biol Chem 2006;281:34696-704.

43. Wang Z, Park HJ, Carr JR, et al. FoxM1 in tumorigenicity of the neuroblastoma cells and renewal of the neural progenitors. Cancer Res 2011;71:4292-302.

44. Ochiai H, Takenobu H, Nakagawa A, et al. Bmil is a MYCN target gene that regulates tumorigenesis through repression of KIF1Bbeta and TSLC1 in neuroblastoma. Oncogene 2010;29:2681-90. 